\title{
Pretibial epidermolysis bullosa: genetic linkage to COL7A1 and identification of a glycine-to-cysteine substitution in the triple-helical domain of type VII collagen
}

\author{
Angela M.Christiano', Julia Yu-Yun Lee ${ }^{3}$, Wei J.Chen ${ }^{4}$, Sal LaForgia ${ }^{1}$ and Jouni Uitto, ${ }^{1,2 *}$ \\ Departments of ${ }^{1}$ Dermatology, and ${ }^{2}$ Biochemistry and Molecular Biology, Jefferson Medical College, and Section of Molecular Dermatology, \\ Jefferson Institute of Molecular Medicine, Thomas Jefferson University, Philadelphia, PA, USA, ${ }^{3}$ Department of Dermatology, National Cheng- \\ Kung University Medical Center, Taiwan and ${ }^{4}$ Institute of Epidemiology, College of Public Health, National Taiwan University, Taipei, Taiwan, \\ ROC
}

Received March 31, 1995; Revised and Accepted June 5, 1995

Pretibial epidermolysis bullosa (PEB) is a rare variant of dominant dystrophic EB (DDEB) in which recurrent blistering with scarring predominantly involves the pretibial skin. Although blistering appears to be localized clinically, electron microscopy of the dermalepidermal junction in patients with PEB reveals anchoring fibril abnormalities that are not restricted to the predilection sites. Furthermore, PEB cannot be distinguished from the generalized (CockayneTouraine and Pasini) types of DDEB on the basis of anchoring fibril morphology alone. The generalized forms of DDEB have been linked to the type VII collagen gene (COL7A1) on chromosome 3p21. In this study, we sought to test the hypothesis that mutations underlying PEB also reside in COL7A1. We initiated mutational analysis in COL7A1 in a large five-generation PEB family of Taiwanese descent. We identified a G-to-T transversion at nt position 7867, which results in a glycine-to-cysteine substitution (G2623C) in exon 105. This mutation was confirmed in affected family members using the loss of a Smal restriction site, and when used for linkage analysis, together with an intragenic Pvull polymorphism and several flanking markers, resulted in a LOD score of $Z=3.61$ at $\theta=0$ in this family. This is the first demonstration of genetic linkage and mutation analysis in PEB, and illustrates that the CockayneTouraine, Pasini, and now the pretibial clinical variants of DDEB are allelic, resulting from different glycine substitution mutations in the type VII collagen gene.

\section{INTRODUCTION}

Pretibial epidermolysis bullosa (PEB) is a rare variant of dominant dystrophic EB (DDEB) in which recurrent blistering and scarring predominantly involve the pretibial skin, with variable nail dystrophy, hypertrophic scarring and albopapuloid skin lesions $(1-4)$. Although blistering appears to be localized clinically, electron microscopy of the dermal-epidermal junction in patients with PEB has revealed anchoring fibril abnormalities that are not restricted to the predilection sites $(2,3)$. Furthermore, using morphometric analysis of the anchoring fibrils, we have demonstrated that PEB cannot be distinguished from the other variants of DDEB, such as the CockayneTouraine and the Pasini types, on the basis of anchoring fibril abnormalities (2). Thus, PEB represents a rare, yet interesting, localized clinical variant which may be allelic with other forms of DDEB. This possibility is also supported by the observation of family members with the Pasini or Cockayne-Touraine type of clinical presentation in some PEB kindreds $(2,4)$.

Families with DDEB have recently been linked to the type VII collagen gene (COL7A1) (5-8) which resides on chromosome 3p21 $(9,10)$. In fact, a total of 14 families with a combined maximum LOD score $(Z)$ of 41.4 at $\theta=0$ have been recorded thus far, with no evidence for locus heterogeneity (11). In this study, we provide evidence for linkage of PEB to COL7Al in a large three-generation family of Taiwanese descent. Furthermore, we demonstrate a pathogenetic glycine substitution mutation in the triple helical region of the type VII collagen gene which co-segregates with the PEB phenotype in this family.

\section{RESULTS}

Clinical and genetic features

The family subjected to study consisted of 12 affected living individuals in three generations (Fig. 1). The clinical phenotype was characterized by pretibial blisters which developed into prurigo-like hyperkeratotic lesions (Fig. 2). Strikingly, the lesions were present predominantly on the pretibial areas, sparing the knees and other parts of the skin (Fig. 2). Other clinical features included nail dystrophy, albopapuloid skin lesions, and hypertrophic scars without pretibial predominance. There was considerable inter-individual variability which has been detailed in a previous report (2) (see Clinical description

*To whom correspondence should be addressed 


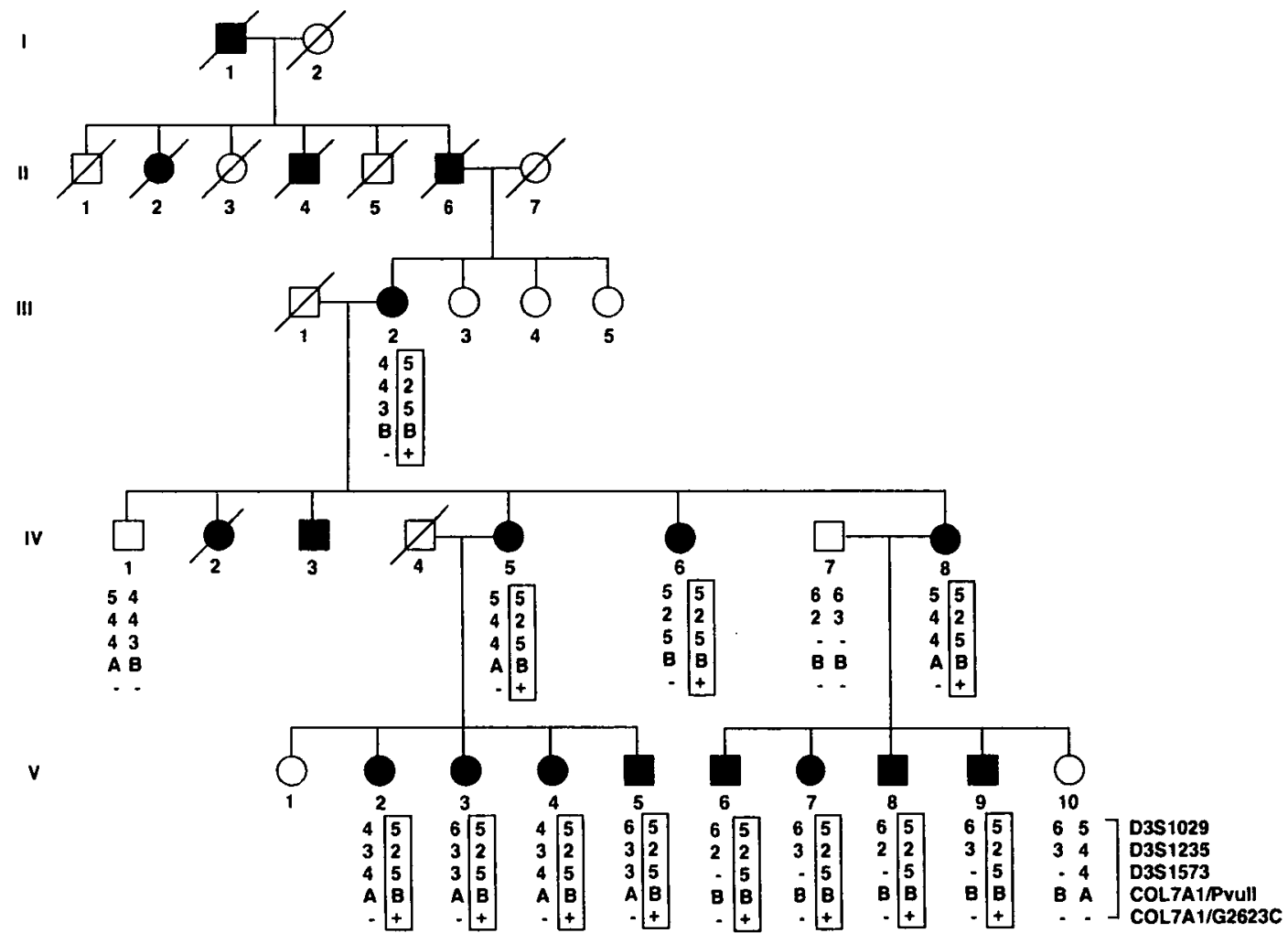

Figure 1. Pedigree of the family with pretibial epidermolysis bullosa (PEB). Haplotype analysis was performed on 12 affected and two unaffected family members representing three generations, and the genotypes were established with markers shown on the right of generation V. The anonymous markers D3S 1029 , D3S1235, and D3S1573 are microsatellites flanking the COL7A1 locus at 3p21. The marker D3S1029 is telomeric, while the exact relative position of the two other microsatellites with respect to the COL7A1 locus has not been established. The COL7Al/PvuII polymorphism represents an intragenic silent single-base substitution. COL7A1/G2623C is the mutation co-segregating with the clinical phenotype. Co-inheritance of the clinical phenotype with the boxed haplotype is noted, with $Z=3.61$ at $\theta=0$.

in Materials and Methods). Light microscopy of the pretibial lesions showed sub-epidermal blister formation, with fibrosis and vascular proliferation in the floor of each blister. Ultrastructurally, all lesions showed dermal-epidermal separation beneath the lamina densa. The anchoring fibrils were rudimentary in both lesional and non-lesional skin.

\section{Genetic linkage analysis}

Recent cloning of the human type VII collagen gene (COL7A1) $(12,13)$ and its mapping to $3 p 21(9,10)$ allowed us to perform genetic linkage analyses in families with dystrophic EB. In this study, an intragenic PvuII polymorphism was initially used for linkage analysis $(5,14)$. This marker was partially informative in this family yielding a maximum LOD score $(Z)$ of 1.75 at $\theta=0$ (Table 1). Subsequently, three flanking markers (D3S1029, D3S1235, and D3S1573) (15) were partially informative with no recombination events (Table 1). The data were consistent with genetic linkage of the PEB locus to COL7A1 in this family. This conclusion was subsequently verified by demonstration of a single base substitution in COL7A1 (see below), and genetic linkage of the mutation with the PEB phenotype resulted in a maximum LOD score $(Z)=3.61$ at $\theta=0$. Several three- or four-point linkage analyses (assuming the marker intervals being known; see Table 1) were performed with the LINKMAP program. Once the mutation was included in the analyses, the maximum LOD scores between the PEB and the mutation as well as the four informative markers were very similar, ranging from 3.60 to 3.61. No recombination between any of the markers and the mutation was observed.

\section{Mutation identification}

During verification of a different missense mutation in one allele in COL7A1 of an unrelated proband with recessive DEB (R2622Q) which resulted in loss of an Smal site (CCCGGG to CCCAGG), two affected individuals in the family with PEB reported in this study also showed a similar restriction digestion pattern. To examine the basis of this restriction enzyme site change, exons 105 and 106 of COL7A1 were PCR amplified from the flanking intronic sequences, and the product was subjected to direct nucleotide sequencing. The results revealed a G-to-T transversion in nt position 7867 of the type VII collagen cDNA (Fig. 3). This substitution resulted in a change of a glycine (GGC) to a cysteine (TGC) codon, and the mutation was designated as $\mathrm{G} 2623 \mathrm{C}$. As indicated above, this mutation resulted in a loss of a SmaI restriction enzyme site (CCCGGG to CCCGGI), and the inheritance of the mutation in the family was confirmed at the DNA level by digestion with this restriction enzyme (Fig. 3). Examination of 78 unrelated individuals with different forms of $\mathrm{EB}$, and 34 


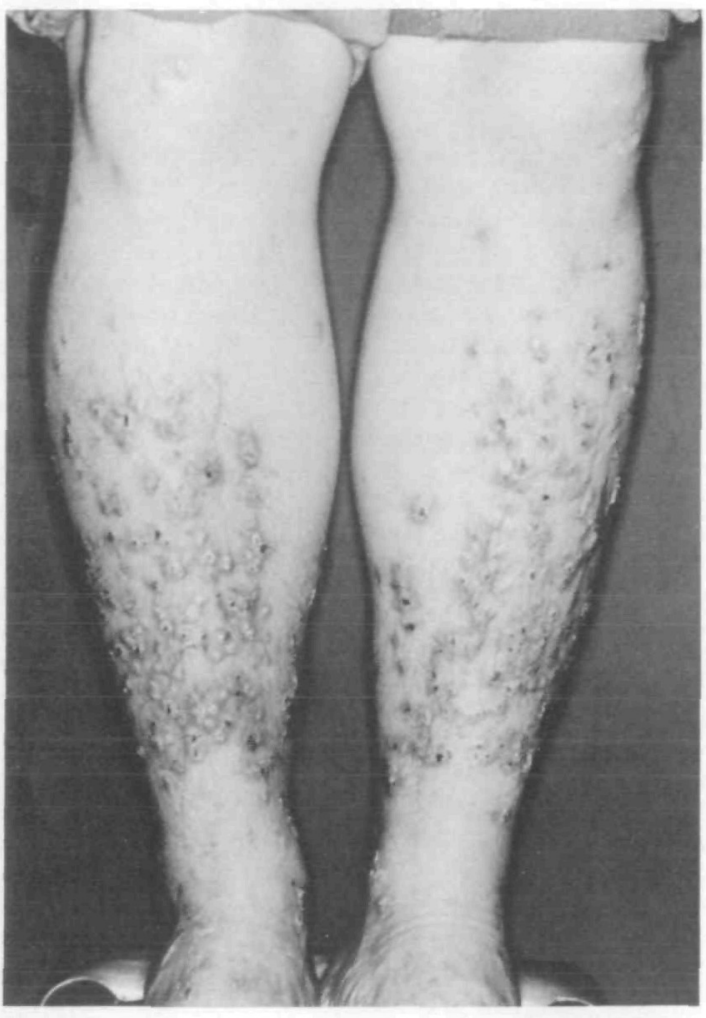

Figure 2. Clinical presentation of a family member with PEB (V-8 in Fig. 1). Note the presence of prurigo-like cutaneous lesions with excoriation, largely limited to the pretibial area.

unrelated, unaffected control individuals, did not reveal the same loss of the restriction enzyme site (with the exception of the family with the R2622Q mutation; see above). Thus, the mutation detected in the family with PEB, G2623C, is not a common polymorphism, nor is it a common mutation among the patients with the dystrophic forms of EB. Also, as indicated above, this mutation completely co-segregated with the clinical phenotype in this family, including those manifesting with nail dystrophy only, and one member showing albopapuloid PEB.

\section{DISCUSSION}

In this study, we have demonstrated that a single base change resulting in a glycine-to-cysteine substitution in the triple helical domain of type VII collagen co-segregates with the phenotype in a family with a dominantly inherited form of DEB, with unique clinical features. Specifically, these patients demonstrate localized, predominantly pretibial blister formation, with nail dystrophy and hypertrophic scarring (2). Thus, the clinical phenotype is clearly distinct from the more generalized forms of DDEB, the Cockayne-Touraine and Pasini types (1). It should be noted, however, that the ultrastructural features of the dermal-epidermal junction of the skin in all three forms of DDEB, i.e., sub-basal lamina tissue separation and paucity of anchoring fibrils, are indistinguishable. Furthermore, all three types of DDEB are allelic, since we have previously demonstrated specific mutations in COL7Al in three different families with dominantly inherited DEB with features of the
Table 1. Two-point LOD scores for the pretibial variant of dominant dystrophic epidermolysis bullosa and markers on chromosome 3

\begin{tabular}{|c|c|c|c|c|c|c|c|}
\hline \multirow[t]{2}{*}{ Marker } & \multicolumn{7}{|l|}{$\theta$} \\
\hline & 0 & 0.01 & 0.05 & 0.1 & 0.2 & 0.3 & 0.4 \\
\hline D3S1029 & 0.89 & 0.88 & 0.81 & 0.71 & 0.51 & 0.29 & 0.09 \\
\hline D3S1235 & 1.87 & 1.82 & 1.60 & 1.32 & 0.71 & 0.06 & -0.42 \\
\hline D3S1573 & 1.51 & 1.48 & 1.35 & 1.19 & 0.86 & 0.53 & 0.23 \\
\hline$P v u \mathrm{II}$ & 1.75 & 1.72 & 1.57 & 1.39 & 1.01 & 0.63 & 0.30 \\
\hline G2623C & 3.61 & 3.55 & 3.32 & 3.01 & 2.35 & 1.61 & 0.80 \\
\hline
\end{tabular}

The distances $(\theta)$ between the markers were set at: (telomere)-D3S1029(0.005)-D3S1235-(0.003)-D3S1573-(0.003)-Pvull-(0.003)-G2623C-(0.006)(centromere)

A.
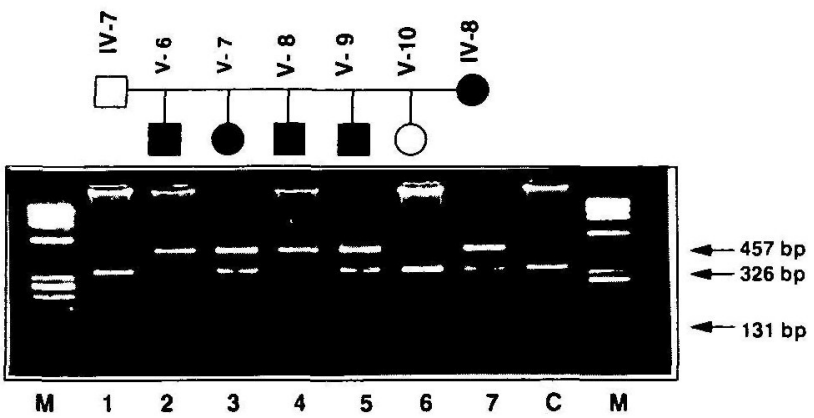

B.
G A T C

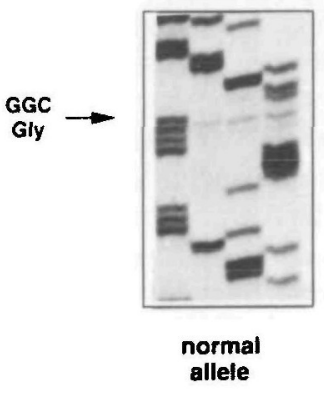

G A T C

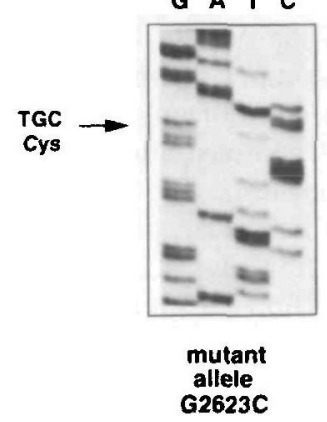

Figure 3. Mutation detection in the family with PEB. (A) Digestion of PCR products representing exons 105-106 of COL7Al with SmaI from representative individuals of the family shown in Fig. 1. The nucleotide substitution (see below) resulted in the loss of a naturally occurring SmaI site. Thus, in normal individuals, the $457 \mathrm{bp} \mathrm{PCR} \mathrm{product} \mathrm{is} \mathrm{completely} \mathrm{digested}$ to 326 and $131 \mathrm{bp}$ fragments. In the affected individuals, heterozygous for the nucleotide substitution, an undigested $457 \mathrm{bp}$ band, in addition to the digestion fragments, is noted. (B) Sequencing of the PCR product containing the mutation reveals the presence of a $T$, instead of a $G$ noted in the normal allele. This nucleotide substitution results in glycine (GGC) substitution by a cysteine (TGC); this mutation is designated as G2326C.

Cockayne-Touraine or Pasini type (16-18). It should be noted that another dominantly inherited clinical variant of dystrophic $\mathrm{EB}$, the Bart syndrome, has also been linked to the COL7A1 locus (19). Thus, four different subtypes of DDEB studied thus far appear to be due to mutations in the COL7A1 gene.

The mutation disclosed in the family with PEB, G2623C, replaces a glycine residue in a collagenous domain consisting 
of 74 uninterrupted Gly-X-Y repeats. This mutation creates a new cysteine residue 11 amino acids upstream from a conserved cysteine residue in COL7A1 (C2634), which is thought to play a critical role in intermolecular assembly of type VII collagen molecules into anchoring fibrils (20). The previously disclosed dominant dystrophic EB mutations consist of glycine substitutions (G2040S, G2043R and G2351R) (16-18) which reside upstream from the mutation described in this family with PEB. All these substitutions affect different glycine residues in the collagenous sequence of type VII collagen.

The new cysteine residue generated by the mutation in the PEB family could potentially result in the formation of an abnormal disulfide bond. Alternatively, the substitution of a glycine in the collagenous (Gly-X-Y) ${ }_{n}$ sequences could destabilize the critical triple-helical conformation of the collagenous domain. Glycine substitutions in other collagen genes have been shown to be the basis for a variety of heritable connective tissue diseases. Specifically, mutations in collagens type I, II, III, and IV, largely consisting of glycine substitutions within the triple helical domain of the protein, have been shown to be the basis of osteogenesis imperfecta, chondrodystrophies, Ehlers-Danlos syndrome IV, and Alport syndrome, respectively (21-23). Thus, the likely explanation for the fragility of the skin in the PEB family is abnormalities in anchoring fibrils due to destabilization of the type VII collagen triple helical domain as a result of glycine substitution. It should be noted that since type VII collagen is a homotrimer of three identical $\alpha 1$ (VII) chains, one out of eight molecules will consist of three normal polypeptides, assuming equal expression of both alleles at the protein level (24). Thus, one would expect that a small number of anchoring fibrils can be assembled from these normal molecules, an observation consistent with ultrastructural demonstration of the presence of a few, often thin anchoring fibrils. The presence of some anchoring fibrils may explain the milder phenotype of DDEB, in contrast to the extreme fragility of the skin observed in the recessively inherited forms, such as the Hallopeau-Siemens type of DEB, which demonstrate a complete absence of anchoring fibrils in most cases due to the presence of premature termination codons in both COL7Al alleles (25-28).

\section{MATERIALS AND METHODS}

\footnotetext{
Clinical description

The family under study was Family $C$ from a five-generation kindred of PEB reported previously (2). The proband was a 47 year old Taiwanese woman (IV-8, Fig. 1). She presented with a history of blistering over the pretibial areas resulting in hypertrophic scars and milia. Her big toe nails were lost since early childhood after trauma. The mucous membranes were not involved. Histopathology of two skin lesions from the shins revealed subepidermal blister or cleft formation. The anchoring fibrils were found to be sparse and rudimentary or absent in two affected members from another branch of the family with PEB (Family E, in ref. 2). The clinicopathologic features of the affected family members were consistent with PEB. One of the proband's sons (V-8, Fig. 1) had prurigo-like papules on the extensor aspects of the extremities, most extensive on the shins (Fig. 2). Albopapuloid lesions were noted on the trunk. Except for the left ring finger, all the nails were either lost or dystrophic. This individual was diagnosed as having 'albopapuloid PEB'. The proband's remaining three affected children ( $\mathrm{V}-6,7,9)$ manifested only with nail changes since childhood. The other affected members related to Family C (III-2, IV-3,5,6 and V-2-5, Fig. 1) showed nail atrophy with mild or absent pretibial lesions. In an initial report, individuals V-2 to V-5 were anecdotally reported to have been unaffected (2). However, upon examination of family members by one of the authors (J.Y.-Y.L.), V.2 to V-5 were found
}

to have nail dystrophy since childhood, and were therefore considered to be affected.

\section{Linkage studies}

DNA markers used in linkage analysis include an intragenic marker ( $P v u I I)$ $(5,14)$, and three flanking markers which map to 3p21 (D3S1573, D3SI235, D3S1029) (15). These markers were included based on physical mapping data which place them in close proximity to the COL7Al locus (S.L., unpublished results). All markers were assayed by PCR amplification of genomic DNA using specific primers based on intronic sequences (PvuII) (14) or obtained from the Genome Data Base (D3S1573, D3S1235, D3S1029) (15). PCR reactions were carried out in a total volume of $50 \mu \mathrm{l}$ with $250 \mathrm{ng}$ of genomic DNA, $40 \mathrm{ng}$ of each primer, $250 \mu \mathrm{M}$ of each dNTP and $2.5 \mathrm{U}$ AmpliTaq DNA polymerase in $1 \times$ reaction buffer supplied by the manufacturer (PerkinElmer Cetus). The reaction cycle was optimized for each primer pair. In general, DNA was initially denatured for $5 \mathrm{~min}$, followed by 40 cycles of denaturation $\left(94^{\circ} \mathrm{C}\right.$ for $\left.45 \mathrm{~s}\right)$; annealing $\left(55-60^{\circ} \mathrm{C}\right.$ for $\left.45 \mathrm{~s}\right)$; and extension $\left(72^{\circ} \mathrm{C}\right.$ for $\left.60 \mathrm{~s}\right)$. After amplification, the product containing the intragenic polymorphism was digested with the restriction endonuclease, PvuII, and fractionated on a $2 \%$ agarose gel to separate the two alleles. To distinguish the alleles of D3S1573, D3S1235, and D3S1029, the specific primers were end-labelled prior to PCR amplification, the amplified products were then fractionated on an $8 \%$ polyacrylamide gel, and the gel was vacuum dried and subjected to autoradiography.

Linkage analysis was performed assuming autosomal dominant inheritance of PEB, an allelic frequency of 0.003 for the PEB allele, and equal recombination rates for males and females. Phenocopy and mutation rates were set at zero. The allelic frequencies of DNA markers were estimated from 37 unrelated individuals. The two-point and multipoint linkage analyses were carried out using the MLINK and LINKMAP programs from LINKAGE, respectively (version 5.1) (29).

\section{Mutation identification}

The 457 bp region of the COL7Al gene containing exons 105-106 was PCR amplified using the primers: Left, 5'GGCGATTCTCTTTGGTCCCT3'; and right, $5^{\prime}$ GCAGTGGGGTGAGCCTTAGG3'. Following denaturation at $94^{\circ} \mathrm{C}$ for $5 \mathrm{~min}$, the cycling conditions were $94^{\circ} \mathrm{C}$ for $45 \mathrm{~s} ; 57^{\circ} \mathrm{C}$ for $45 \mathrm{~s} ; 72^{\circ} \mathrm{C}$ for $45 \mathrm{~s}$, for 40 cycles. PCR products were verified on $2 \%$ agarose gels and purified in a QIAquick PCR purification column (QIAgen) followed by direct sequencing on an automated sequencing system (Applied Biosystems, Inc.), or subcloning into the TA vector followed by manual sequencing (InVitrogen). Digestion of the PCR products was performed with the restriction endonuclease Smal according to the manufacturer's recommendations (New England Biolabs) and fractionated on $3 \%$ agarose gels. The presence of the mutant allele resulted in the loss of a Smal site, yielding an undigested band of 457 bp, in addition to the two restricted fragments of 326 and 131 bp in affected individuals heterozygous for the mutation G2623C. In unaffected control individuals, only the two digestion products were observed.

\section{ACKNOWLEDGEMENTS}

We appreciate the excellent technical assistance of Yili $\mathrm{Xu}$ and $\mathrm{Xin}$ Zhang, and thank Tamara Alexander and Eileen O'Shaughnessy for preparation of the manuscript. We appreciate the interest and participation of the family members in this study. This work was supported in part by USPHS, NIH Grant PO1-AR38923, the Dermatology Foundation, the March of Dimes Birth Defects Foundation, the Lin Roug-San Culture and Public Welfare Foundation, and Taiwan National Science Council Grant 82-0412-B006-093-M02.

\section{REFERENCES}

1. Fine, J.D., Bauer, E.A., Briggaman, R.A., Carter, D.M., Eady, R.A., Esterly, N.B., Holbrook, K. A., Hurwitz, S., Johnson, L., Lin, A., Pearson, R., Sybert, V.P. (1991) Revised clinical and laboratory criteria for subtypes of inherited epidermolysis bullosa: A consensus report by the subcommittee on diagnosis and classification of the national epidermolysis bullosa registry. J. Am. Acad. Dermatol. 24. 119-153.

2. Lee, J.Y.Y., Chen, H.C., Lin, S.J. (1993) Pretibial epidermolysis bullosa: A clinicopathologic study. J. Am. Acad. Dermatol. 29, 974-981.

3. Lichtenwald, D.J., Hanna, W., Sauder, D.N., Jakubovic, H.R., Rosenthal, D. (1990) Pretibial epidermolysis bullosa: report of a case. J. Am. Acad. Dermatol. 22, 346-350. 
4. Garcia-Perez A., Carapeto F.J. (1975) Pretibial epidermolysis bullosa: report of two families and a review of the literature. Dermarologica 150 , $122-128$.

5. Ryynänen, M., Knowlton, R.G., Parente, M.G., Chung, L.C., Chu, M.-L., Uitto, J. (1991) Genetic linkage of the gene (COL7A I) on chromosome 3 to dominant dystrophic epidermolysis bullosa. Am. J. Hum. Genet. 49, 797-803.

6. Ryynänen, M., Ryynänen, J., Sollberg, S., Iozzo, R.V., Knowlton, R.G., Uitto, J. (1992) Genetic linkage of type VII collagen (COL7A1) to dominant dystrophic epidermolysis bullosa in families with abnormal anchoring fibrils. J. Clin. Invest. 89, 974-980.

7. Gruis, N.A., Bavinck, J.N.B., Steijlen, P.M., van der Schroeff, J.C., van Haeringer, A., Happle, R., Mariman, E., Beersum, S.E.C. van, Uitto, J., Vermeer, B.J., Frants, R.R. (1992) Genetic linkage between the collagen VII (COL7Al) gene and the autosomal dominant form of dystrophic epidermolysis bullosa in two Dutch kindreds. J. Invest. Dermatol. 99, $528-530$.

8. Uitto, J., Christiano, A.M. (1993) Dystrophic forms of epidermolysis bullosa. Semin. Dermatol. 12, 191-201.

9. Parente, M.G., Chung, L.C., Ryynänen, J., Woodley, D.T., Wynn, K.C., Bauer, E.A., Mattei, M.-G., Chu, M.-L., Uitto, J. (1991) Type VII collagen: cDNA cloning and chromosomal mapping of the gene. Proc. Natl Acad. Sci. USA 88, 6931-6935.

10. Greenspan, D.S., Byers, M.G., Eddy, R.L., Hoffman, G.G., Shows, T.B. (1993) Localization of the human collagen gene COL7AI to $3 p 21.3$ by fluorescence in situ hybridization. Cytogenet. Cell Genet. 62, 35-36.

11. Uitto, J., Christiano, A.M. (1994) Molecular basis of the dystrophic forms of epidermolysis bullosa: Mutations in the type VII collagen gene. Arch. Derm. Res. 287, 16-22.

12. Christiano, A.M., Greenspan, D.S., Lee, S., Uitto, J. (1994) Cloning of human type VII collagen: Complete primary sequence of the $\alpha$ (VII) chain and identification of intragenic polymorphisms. J. Biol. Chem. 269, 20256-20262.

13. Christiano, A.M., Hoffman, G.G., Chung-Honet, L.C., Lee, S., Cheng, W., Uitto, J., Greenspan, D.S. (1994) Structural organization of the human type VIl collagen gene (COL7A1), comprised of more exons than any previously characterized gene. Genomics 21, 169-179.

14. Christiano, A.M., Chung-Honet, L.C., Hovnanian, A., Uitto, J. (1992) PCR-based detection of two exonic polymorphisms in the human type VII collagen gene (COL7A1) at 3p21.1. Genomics 14, 827-828.

15. Naylor, S.L., Buys, C.H.C.M., Carritt, B. (1994) Report of the Fourth International Workshop on Human Chromosome 3 Mapping 1993. Cytogenet. Cell. Genet. 65, 2-50.

16. Christiano, A.M., Ryynänen, M., Uitto, J. (1994) Dominant dystrophic epidermolysis bullosa: Identification of a glycine-to-serine substitution in the triple-helical domain of type VII collagen. Proc. Natl Acad. Sci. USA 91, 3549-3553.

17. Christiano, A.M., Morricone, A., Paradisi, M., Angelo, C., Mazzanti, C., Cavalieri, R., Uitto, J. (1995) Dominant dystrophic epidermolysis bullosa: A glycine-to-arginine substitution in the triple-helical domain of the type VII collagen gene. J. Invest. Dermatol. 104, 438-440.

18. Burgeson, R.E., Anton-Lamprecht, I., Christiano, A.M., Ebschner, U., Amano, S., Uitto, J. (1995) Compound heterozygosity for COL7A1 mutations in twins with dystrophic epidermolysis bullosa: A maternal glycine substitution and a paternal insertion/deletion result in a severe recessive phenotype. J. Invest. Dermatol. 104, 582 (abstract).

19. Zelickson, B., Matsumura, K., Kist, D., Epstein, E.H., Bart, B.J. (1995) Bart's Syndrome: ultrastructure and genetic linkage. Arch. Dermatol. 131, 663-668.

20. Burgeson, R.E. (1993) Type VII collagen, anchoring fibrils, and epidermolysis bullosa. J. Invest. Dermatol. 101, 252-255.

21. Christiano, A.M., Greenspan, D.S., Hoffman, G.G., Zhang, X., Tamai, Y., Lin, A.N., Dietz, H.C., Hovnanian, A., Uitto, J. (1993) A missense mutation in the human type VII collagen gene in two siblings with recessive dystrophic dystrophic epidermolysis bullosa. Nature Genet. 4, 62-66.

22. Kivirikko, K.I. (1993) Collagens and their abnormalities in a wide spectrum of diseases. Ann. Med. 25, 113-126.

23. Hudson, B.G., Reeders, S.T., Tryggvason, K. (1993) Type IV collagen: structure, gene organization, and role in human diseases. J. Biol. Chem. 268, 26033-26036.

24. Stolle, C.A., Pyeritz, R.E., Myers, J.C., Prockop, D.J. (1985) Synthesis of an altered type III procollagen in a patient with type IV Ehlers-Danlos syndrome. J. Biol. Chem. 260, 1937-1944.
25. Christiano, A.M., Anhalt, G., Gibbons, S., Bauer, E.A., Uitto, J. (1994) Premature termination codons in the type VII collagen gene (COL7A1) underlie severe, mutilating recessive dystrophic epidermolysis bullosa. Genomics 21, 160-168.

26. Hilal, L., Rochat, A., Duquesnoy, P., Blanchet-Bardon, D., Wechsler, J., Martin, N., Christiano, A.M., Barrandon, Y., Uitto, J., Goossens, M., Hovnanian, A. (1993) A homozygous frameshift mutation in COL7Al predicting a shortened protein in the generalized mutilating (HallopeauSiemens) form of recessive dystrophic epidermolysis bullosa. Nature Genet. 5, 287-293.

27. Hovnanian, A., Hilal, L., Blanchet-Bardon, C., de Prost, Y., Christiano, A.M., Uitto, J., Goossens, M. (1994) Recurtent nonsense mutations within type VII collagen in patients with severe mutilating recessive dystrophic epidermolysis bullosa. Am. J. Hum. Genet. 55, 289-296.

28. Christiano, A.M., Suga, Y., Greenspan, D.S., Ogawa, H., Uitto, J. (1995) Premature termination codons on both alleles of the type VII collagen gene (COL7Al) in three brothers with recessive dystrophic epidermolysis bullosa. J. Clin. Invest. 95, 1328-1334.

29. Lathrop, G.M., Lalouel, J.M., Juller, C., Ott, J. (1984) Strategies for multilocus linkage analysis in humans. Proc. Natl Acad. Sci. USA 81, 3443-3446. 